\title{
Propranolol significantly reduced DNA polymerase $\beta$ expression in patients with essential tremor
}

\author{
Nefise Kandemir ${ }^{1,2}$, Sercan Kenanoglu ${ }^{1}$, Murat Gultekin ${ }^{3}$, Nuriye Gokce ${ }^{1}$, \\ Hilal Akalin ${ }^{1,4}$, Nazife Tascioglu ${ }^{1}$, Meral Mirza ${ }^{3}$, Emel Koseoglu ${ }^{3}$, and Munis Dundar ${ }^{1 *}$
}

ABSTRACT

\section{BACKGROUND}

Essential tremor (ET) is the most common movement disorder. Propranolol is a first-line medication for ET. We aimed to evaluate the effect of propranolol on the expression of poly (ADP-ribose) polymerase 1 (PARP1) and DNA polymerase beta $(P O L B)$ genes, which are known to be related to neurodegenerative diseases, in patients with ET.

\section{METHODS}

Thirty-five healthy volunteers and thirty-five patients followed up with essential tremors were included in a non-randomized control experimental study. Expressions of $P A R P 1$ and $P O L B$ genes were compared between the control group and the patient group. In addition, pre- and post-treatment gene expression levels and Fahn-Tolosa-Marin tremor scale values of the patient group were compared after 8 weeks of propranolol treatment. The Wilcoxon rank and Mann Whitney U tests were used to analyze the data.

\section{RESULTS}

At baseline, PARP1 expression was significantly lower in the ET group than in the control group. ( $\mathrm{p}<0.001)$. POLB gene expression was significantly higher in the pre-treatment ET group than in the controls $(\mathrm{p}<0.05)$. There was no significant difference in $P A R P 1$ expression levels before and after 8 weeks of propranolol treatment. $P O L B$ gene expression was significantly higher in the pre-treatment group than in the post-treatment group $(\mathrm{p}<0.001)$.

\section{CONCLUSION}

Propranolol significantly decreased $P O L B$ gene expression but there was no significant difference in $P A R P 1$ gene expression levels in the patient group, after 8 weeks of propranolol treatment.

Keywords: Tremor, genes, propranolol, essential tremor
${ }^{1}$ Department of Medical Genetics, Erciyes University Faculty of Medicine, Kayseri, Turkey

${ }^{2}$ Department of Medical Genetics, Diskapi Yildirim Beyazit Training and Research Hospital, Ankara, Turkey

${ }^{3}$ Department of Neurology, Erciyes University Faculty of Medicine, Kayseri, Turkey

${ }^{4}$ Department of Medical Genetics, Ahi Evran University Faculty of Medicine, Kirsehir, Turkey

*Correspondence:

Munis Dundar, Professor

Erciyes University Medical School, Department of Medical Genetic,

38039 Kayseri/Turkey

Mobile: +905326486116

E-mail: dundar@erciyes.edu.tr ORCID ID :0000-0003-0969-4611

Date of first submission, June 21, 2021

Date of final revised submission,

September 29, 2021

Date of acceptance, October 1, 2021

This open access article is distributed under a Creative Commons AttributionNon Commercial-Share Alike 4.0 International License
Cite this article as: Kandemir N,
Kenanoglu S, Gultekin M, et al. Propra-
nolol significantly reduced DNA poly-
merase $\beta$ expression in patients with
essential tremor. Univ Med 202 $1 ; 40$ :
207-15. doi: $10.18051 /$ UnivMed.2021.
v40.207-215 


\section{INTRODUCTION}

Essential tremor (ET) that provokes uncontrolled and rhythmical contractions and relaxations (shaking, twitching movements) is one of the progressive neurologic movement disorders. The disease is also called idiopathic tremor or benign tremor. Even though the disease can occur at any period of life, it is more frequently seen in the older age group. The prevalence of ET has a wide range, from $1 \%$ in the general population to $5 \%$ in the elderly. ${ }^{(1,2)}$ The main motor dysfunction of ET is a kinetic tremor that occurs during voluntary movements of the hands and arms and over time spreads to the head, vocal cords, jaw, and other anatomical parts. Another common sign of ET is bilateral postural tremor. Essential tremor is a clinically heterogeneous disease and also comprises many disorders such as cognitive disorders, dementia and sensory and psychiatric disorders. Age and family history can be considered as major risk factors for ET. ${ }^{(3)}$ The diagnosis is made based on the typical pattern of tremor and the exclusion of other diseases with tremor. Despite its high prevalence, especially in the elderly, the pathophysiology of the disease is still unclear, although many cases seem to be familial. ${ }^{(3-5)}$

There are still several parts in the etiology of ET which need to be clarified but the most known substantial etiological factors are genetic and environmental. Many large family group samples show an autosomal-dominant pattern. First-degree relatives of ET patients have a greater risk of ET and the risk increases if the tremor starts at an early age. ${ }^{(5)}$ Considering that more than half of the affected individuals have a family history, genetics plays a substantial role in the disease. In addition, there is higher concordance in monozygotic twins than in dizygotic twins. ${ }^{(6)}$ Some studies showed that concordance among monozygotic twins is approximately $60 \%$ but reported studies about sporadic cases and variable age of onset in some familial cases are arguments for nongenetic factors. ${ }^{(7)}$ A positive family history is quite frequent in patients with ET, among whom a significant number of families presumably have autosomal dominant inheritance, while patients with a positive family history have a younger age of onset for the disease. ${ }^{(8)}$

Essential tremor and some neurodegenerative diseases such as Parkinson's disease (PD), dystonia, and progressive supranuclear palsy (PSP) overlap in clinical and pathological features. Some post-mortem examinations have shown that there are tau aggregates in brain samples of patients with ET, similar to those in PSP. Besides, it has been reported that tremors are present also in PD as well as in ET. ${ }^{(9)}$

There are several reported responsible genes for the inheritance of the disease, for example, HS1-binding protein-3 (HS1BP3), essential tremor 1-4 (ETM1, ETM2, ETM3, ETM4), dopamine receptor $\mathrm{D} 3(D R D 3)$, glial high-affinity glutamate transporter member 2 (SLC1A2), hightemperature requirement $\mathrm{A}$ serine peptidase 2 (HTRA2), fused in sarcoma (FUS), leucine-rich repeat and Ig domain containing nogo receptorinteracting protein 1 (LINGO1), and teneurin transmembrane protein 4 (TENM4). ${ }^{10,11)}$

Patients with ET have medical and surgical treatment options. Propranolol, primidone, topiramate, and gabapentin are generally first choice medicines and another treatment option is surgery that includes thalamotomy, tumoral surgery, and deep brain stimulation. ${ }^{(12)}$

Poly (ADP-ribose) polymerase 1 (PARPl) is an ADP-ribosylating enzyme essential for initiating various forms of DNA repair. In the pathological condition, extensive DNA damage in cells results in PARPI cleavage (inactive), preventing DNA repair and thereby leading to cell apoptosis or necrosis. ${ }^{(13,14)}$

Poly (ADP-ribose) polymerase 1 is a gene encoding the poly (ADP-ribosyl) transferase enzyme and is localized in human chromosome 1 (1q42.12). PARP1 encoded by this gene can be activated by DNA damage; it is a nuclear enzyme involved in DNA repair, genomic stability, and many physiological processes such as cell 
apoptosis. PARPI can act as a sensor for DNA damage. After binding to damaged DNA, PARPI binds covalently to nuclear acceptor proteins by forming homodimers and catalyzes the cleavage of NAD + to nicotinamide and ADP-ribose to synthesize long-branching poly (ADP ribose) polymers. ${ }^{(15)}$

The DNA polymerase beta (POLB) is a gene localized on the 8 th chromosome $8 \mathrm{p} 11.21$ region, and is an important type of DNA polymerase that participates in gap-filling DNA synthesis. The protein is normally found in the cytoplasm and functions as a monomer but migrates to the nucleus in case of DNA damage. ${ }^{(16)}$

In the literature, many studies observed changes in the expression levels of PARPI and $P O L B$ genes in diseases with pathological features like those of ET. However, no studies have been conducted to evaluate the effect of propranolol on $P A R P 1$ and $P O L B$ genes in ET patients.

In this study we therefore investigated the effect of propranolol on $P A R P 1$ and $P O L B$ genes in patients with essential tremors (ET), which are known to be related to neurodegenerative diseases.

\section{METHODS}

\section{Research design}

This was a non-randomized control experimental study that was conducted on 35 patients diagnosed with ET in Erciyes University Medical Genetics and Neurology Departments between July 2016 and January 2019.

\section{Research subjects and intervention}

Thirty-five healthy volunteers and thirty-five patients who were followed up with essential tremors were included in the study after they were assessed by the Erciyes University Adult Neurology and Medical Genetics Departments. Volunteers and patients were assessed by neurological examination and the Fahn-TolosaMarin Clinical Rating Scale. As a result, those without neurological disorders or chronic medical diseases were selected as the control group. Power analysis was performed for 35 patients and 35 healthy volunteers to determine sample size. The effect size was 0.082 , 1 -beta was 0.924 and alpha 0.05 .

The inclusion criteria of ET patients were age between 18 and 60 years, diagnosed with ET by a specialist neurologist, having no additional disease and receiving $40 \mathrm{mg}$ propranolol twice a day for eight weeks. Exclusion criteria include patients with hyperthyroidism, diabetes mellitus, psychiatric diseases, tremors (neuropathic, dystonic, and orthostatic), persons receiving treatment that increases tremor and patients under the age of 18 .

The control group consisted of volunteers aged between 18 and 60 who were medically healthy, did not have any neurological diagnosis and did not have a chronic disease, as a result of the evaluation made by a specialist neurologist.

\section{Measurements}

The Fahn-Tolosa-Marin Clinical Rating Scale for Tremor (FTM) was used to assess the essential tremor. ${ }^{(17)}$ Fahn-Tolosa-Marin is a scale used to evaluate resting, postural and action tremors. There are up to five ratings representing its severity. Tremor severity is evaluated by giving an FTM score between 0 and 4. Briefly, the FTM procedure is as follows: the patients are given a device which they hold for 10 seconds to define the tremor severity and frequency. The degree of the scale is directly proportional to the severity of the disease. FTM was measured only in the patient group before and after propranolol treatment, and the two groups were compared statistically.

\section{Laboratory analysis}

After peripheral blood samples had been taken from each of the patients and healthy volunteers into $10 \mathrm{ml}$ EDTA tubes, leukocyte isolation was performed in the laboratory of the Erciyes University Medical Genetics Department with the red cell lysis method. Leukocytes taken 
in TRIzol ${ }^{\mathrm{TM}}$ reagent were stored at $-20^{\circ} \mathrm{C}$ until RNA isolation (Invitrogen, Thermo Fisher Scientific, California, United States). RNA isolation was performed using the phenolchloroform method. cDNA synthesis was done using the EvoScript Universal cDNA Master Mix kit. Expressions of $P A R P 1$ and $P O L B$ genes were performed by using the quantitative real-time PCR method on the Light Cycler 480 II device (Roche Diagnostics Ltd., Rotkreuz, Switzerland) using Real-Time ready Catalog primary-probe kits (PARP1 with assay ID 111143) and POLB with assay ID 147200)) (Roche Diagnostic GmbH, Mannheim, Germany). The reference gene was the $\beta$-Actin housekeeping gene. The expression analyses were performed using the $2^{-\Delta \Delta \mathrm{Ct}}$ formula with Light Cycler 480 software program (version 1.5.0 SP4) (Roche Diagnostics Ltd., Rotkreuz, Switzerland). Gene expression levels were measured in the control group, pre-treatment ET group, and post-treatment ET group (who had received 8 weeks of propranolol treatment).

\section{Statistical analysis}

Statistical analysis was performed with the IBM SPSS Statistics 22 software (IBM SPSS Statistics for Windows, Version22.0. Armonk, NY: IBM Corp. Released 2013). Histograms, Quantile-Quantile Plots, and Shapiro-Wilks tests were used for evaluating the normal distribution of the data. The Wilcoxon rank test was used for pre-post treatment evaluation. Mann Whitney U test was used to evaluate the difference between the control group and the patient group. The Chi-square test was used to compare the distribution according to the gender of the participants. A $p<0.05$ significance level was accepted.

\section{Ethical clearance}

Informed consent was obtained from all subjects who agreed to participate in the study. Ethics committee approval was obtained from Erciyes University Ethical Committee of Clinical Studies (2019/82).

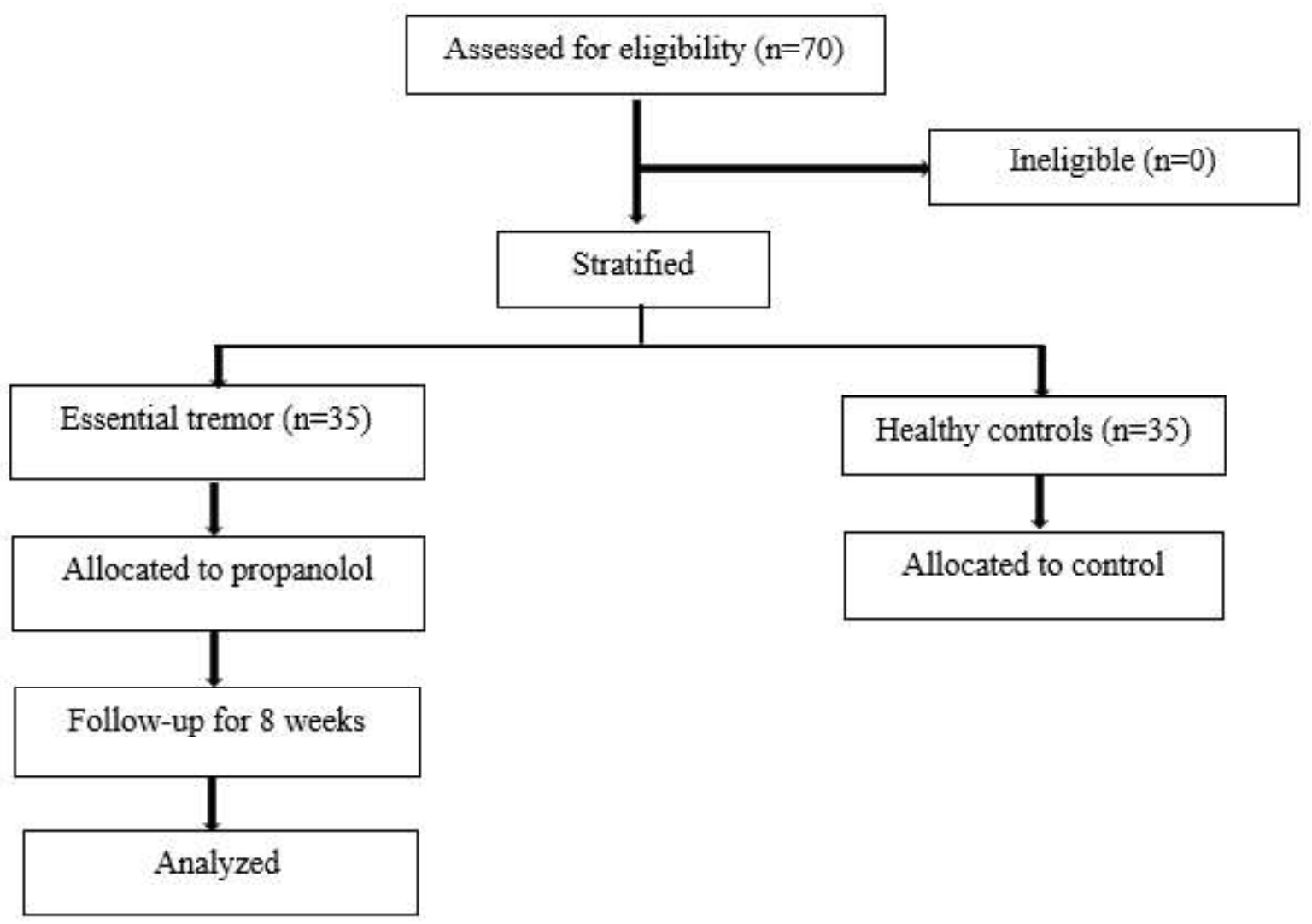

Figure 1. Flow diagram of the participants 
Table 1. Distribution of demographic characteristics, $P A R P 1$ and $P O L B$ at base line

\begin{tabular}{lccc}
\hline Variables & Essential tremor group $(\mathbf{n}=\mathbf{3 5})$ & Control group $(\mathbf{n}=\mathbf{3 5})$ & p value \\
\hline Age (years) & $36.71 \pm 11.55$ & $35.11 \pm 10.59$ & $>0.05^{@}$ \\
Gender & & & \\
Male & 24 & 16 & $>0.05^{\sharp}$ \\
Female & 11 & 19 & \\
$P A R P 1$ & $0.32 \pm 0.18$ & $0.46 \pm 0.16$ & $<0.001^{@}$ \\
$P O L B$ & $0.71 \pm 0.58$ & $0.46 \pm 0.13$ & $<0.05^{@}$ \\
\hline
\end{tabular}

Note: PARP1: poly (ADP-ribose) polymerase 1; POLB: DNA polymerase beta; ${ }^{\circledR}$ Mann-Whitney U test; ${ }^{\#}$ Chi-square test

\section{RESULTS}

Seventy people were stratified into two groups, namely the ET patient group $(\mathrm{n}=35)$ and the healthy control group $(\mathrm{n}=35)$. The ET subjects were given propranolol for 8 weeks (Figure 1). At baseline, there was no significant difference in age and sex (gender) between the ET and control groups (Table 1). A significantly lower PARP1 gene expression level was found in the pretreatment ET group than in the control group $(\mathrm{p}<0.001)$. POLB gene expression was significantly higher in the pre-treatment ET group compared to the control group $(\mathrm{p}<0.05)$ (Table 1). $P O L B$ gene expression was also significantly higher in the pre-treatment group than in the posttreatment group $(\mathrm{p}<0.001)$ (Table 2). Propranolol significantly reduced $P O L B$ expression, but the decrease in PARPl gene expression was not significant.

\section{DISCUSSION}

In our study, the ET patient group showed statistically significant changes in PARP1 and POLB expression levels, in the values compared with the healthy control group. Propranolol significantly reduced $P O L B$ expression, but the reduction in expression of the $P A R P 1$ gene was not significant. Essential tremor, affecting $4 \%$ of individuals aged 40 and over, is one of the most common tremor diseases within the globe. The disease can be either a component of different neurodegenerative diseases or an isolated disease. ${ }^{(18)}$ The molecular mechanism of ET is still unclear because there is no specific diagnostic marker for ET. The disease has clinical similarities with some diseases such as dystonia and Parkinson's disease (PD) and can also be seen together with other neurological diseases such as restless leg syndrome. ${ }^{(19,20)}$ Coexistence with other diseases causes difficulties in the diagnosis of ET.

Twin studies have shown that not only genetic factors, but also environmental factors take part in the etiology of the disease. It was shown in one study that monozygotic twins (6093\%) have greater concordance compared to dizygotic twins $(27-29 \%)$. According to twin studies, the inheritance of ET has been reported to be between $45 \%$ and $90 \% .^{(20,21)}$ The genetic structure of ET is quite complex. The genetic etiology of ET comprises both monogenic and multifactorial inheritance. Only a few regions of sensitivity have been identified in the etiology of ET.

Table 2. PARPI and $P O L B$ expression before and after treatment in the ET group

\begin{tabular}{lccc}
\hline Variables & Before treatment $(\mathbf{n}=\mathbf{3 5})$ & After treatment $(\mathbf{n}=\mathbf{3 5})$ & p value \\
\hline PARP1 & $0.32 \pm 0.18$ & $0.32 \pm 0.21$ & $>0.05^{\$}$ \\
POLB & $0.71 \pm 0.58$ & $0.35 \pm 0.20$ & $<0.001^{\$}$ \\
FTM-TRS Grade & $2.4 \pm 0.9$ & $1.9 \pm 0.7$ & $<0.01^{\$}$ \\
\hline
\end{tabular}

Note: PARP1: poly (ADP-ribose) polymerase 1; POLB: DNA polymerase beta; FTM-TRS: Fahn Tolosa Marin tremor rating scale; ${ }^{\$}$ Wilcoxon rank test 
Genome-wide association studies (GWAS) revealed the complex inheritance of ET. The ETM1, ETM2 and ETM3 genes were found to be more effective in familial ET. On the other hand, the SLC1A2, LINGO1, CTNNA3, PPARGC1A and STK32B genes were observed to be effective in sporadic ET. In whole genome sequencing (WES) studies, rare variants in the FUS, HTRA2, TENM4, NOS3, KCNS2, HAPLN4, USP6 genes have been associated with ET with monogenic inheritance. ${ }^{(22)}$ Liao et al. ${ }^{(23)}$ demonstrated with PheWAS, a phenomewide association study, that propranolol treatment can alter the expression level of genes.

Propranolol is one of the recommended first-line treatment options for essential tremors. This drug is a non-selective beta-adrenergic receptor antagonist and is given in divided doses three times a day. ${ }^{(24)} \beta$-adrenoceptors belong to the family of G-protein-coupled receptors, with the $\beta 1$ and $\beta 2$-adrenoceptors being expressed in the brain. Central $\beta 2$-adrenoreceptor blockage is postulated to have favorable effects on tremors. ${ }^{(25)}$

Poly (ADP-ribose) polymerase-1 (PARPI) has DNA binding and ADP-ribosylation effects. Through these features, PARP 1 constitutes the main part of the cellular repair and defense program mechanism. Poly (ADP-ribose) may also affect some transcription factors, which control the expression of inflammatory mediators, such as nuclear factor kappa B (NFKB). On the other hand, increased PARP1 activation may cause fatal effects on cells by the release of apoptosis-inducing factor (AIF) and $\mathrm{NAD}^{+}$and ATP reduction. Even though PARPl is part of the genome protection mechanisms, hyperactivity of PARP1 can be harmful to the cell. Excessive activation of PARPI has been linked to several biological processes including aging, neurodegeneration, and parthanatosis. Parthanatos is a caspaseindependent programmed cell death pathway because of nuclear translocation of the mitochondria-associated AIF. ${ }^{(26)}$ Excessive activation of PARP1 has been implicated in several neurodegenerative premature aging disorders such as XPA, CSB, and ATM, and the etiology of stroke. ${ }^{(27,28)}$

Poly (ADP-ribose) polymerase-1 has a role in the pathogenesis of Parkinson's disease (PD). This information can be supported by the presence of much PARP in the nuclei of dopaminergic neurons of the substantia nigra in patients with PD. ${ }^{(29)}$ Excessive PARP1 activation, which causes DNA damage, occurs with age in wildtype C. elegans. ${ }^{(28)}$ Multiple studies have shown that increased activation is associated with aging, neurodegeneration, and metabolic disorders. ${ }^{(27)}$ Inhibition of $P A R P I$ provides prominent neuroprotection in animal models; furthermore, it has been determined that PARPI has a physiological role in the regulation of alphasynuclein expression. It has also been shown that variants involved in the PARP1 gene promoter decrease the risk of PD and postpone the initial age of the disease. ${ }^{(30)}$

The ability of PARPI to function simultaneously as both catalytic and acceptor proteins has led to conflicting data. ${ }^{(31)} \alpha$-synuclein $(\alpha$-syn) is a presynaptic protein that accumulates and causes loss of neuronal function in PD. Injection of this protein inhibits $P A R P-1$ activity in the cerebral cortex and hippocampus of rat models. ${ }^{(32-34)}$ The nitric oxide pool that is released after the application of $\alpha$-syn and NO-mediated caspase- 3 activation may play a role in the reduction of $P A R P-1$ activity. ${ }^{(35,36)}$

Both PD and essential tremors are neurodegenerative diseases with tremors. In our results, the expression level of the $P A R P 1$ gene in ET was lower than in the control group. The reason for this result can be related to the fact that PARPI has a significant role in the DNA repair mechanism and the loss of functionality of this gene will be effective in the development of ET. After propranolol treatment, no significant difference was observed in the expression of $P A R P 1$. It is particularly important to conduct studies considering PARP targeted therapy to understand the details of the PARPI catalytic mechanism and regulation. 
DNA polymerase $\beta$ is essential for the base excision DNA repair (BER) pathway, which clears the genome of apurinic/apyrimidinic (AP) regions. ${ }^{(37)}$ Apurinic/apyrimidinic sites could be detrimental for cells because they have mutagenic and/or cytotoxic effects. During the repair of AP regions, DNA pol- $\beta$ participates in synthesis and lyase activities. Due to the crucial role of $P O L B$ in sequence conservation, $P O L B$ is considered critical for cellular survival. The role of $P O L B$ has been confirmed with $P O L B$ null mouse cells, that were more sensitive to agents which damage DNA. ${ }^{(38)}$

Publications are proving that endoreduplication occurs during cell division in patients with PD and Alzheimer's disease (AD). The molecular control mechanisms of endoreduplication in the neurodegeneration process are still confused. Excessive expression of POLB promotes rotenone-induced endoreduplication and this is associated with maintenance of the $\mathrm{G} 2$ state. $P O L B$ is overexpressed during dopaminergic neuronal death, which is associated with cell cycle re-entry in rotenone-based animal models. Therefore, excessive $P O L B$ expression caused by rotenone increases genome instability in dopaminergic cells, through induction of endoreduplication. Increased $P O L B$ and re-entry of the cell cycle may be associated with the loss of dopaminergic neurons. To better understand the relationship between $P O L B$ and endoreduplication, we need more detailed studies, especially using in vivo models. ${ }^{(39)}$

In our study, the $P O L B$ expression level was observed to be higher in ET patients compared to the control group. These results are like those seen in the literature in patients with $\mathrm{AD}$ and PD. After propranolol treatment, the $P O L B$ expression level was lower than in the pre-treatment group. This clearly shows that propranolol treatment reduces the effects of $P O L B$ in ET, considering the recovery status of the patients.

In our study, we examined PARPI and $P O L B$ gene expressions in the blood of ET patients. Considering that ET affects the cerebellar, brain stem, thalamic, and cortical pathways, animal studies can be conducted, and our results can be compared by looking at the expression differences between blood and brain tissue. The number of patients is one of the important limitations of our study. In future studies, looking at the expression levels of these genes with more subjects and in patients treated with different concentrations of propranolol will increase the reliability.

\section{CONCLUSION}

This study demonstrated that $P O L B$ gene expression was significantly higher in the pretreatment group than in the post-treatment group. Gene expression levels and recovery status of patients can be followed by creating groups treated with different propranolol levels, and these are conceived as commencing strategies for emerging novel treatments.

\section{ACKNOWLEDGMENT}

Erciyes University supported the study as project TSA-2019-8891.

\section{CONFLICT OF INTEREST}

The authors declare no competing interests.

\section{CONTRIBUTORS}

NK and MD developed the theory and concept of the study. SK and HA designed the study. MD, MM, MG, and EK investigated and supervised the findings of this work. NK wrote the manuscript with support from NG, NT, SK, and MD. NG and NK performed the statistical analyses. MD, HA and SK verified the analytical methods. NT, NG, and SK made the literature search. MG, MM and EK performed clinical studies. NK analyzed and interpreted the patient data. All authors discussed the results and contributed to the final manuscript. All authors read and approved the final manuscript. 


\section{REFERENCES}

1. Louis ED. Treatment of essential tremor: are there issues we are looking for? Front Neurol 2012;2:18. doi: 10.3389/fneur.2011.00091.

2. Louis ED. Age of onset: can we rely on essential tremor patients to report this? Data from a prospective, longitudinal study. Neuro epidemiology 2013;40:93-8. doi: 10.1159/000341903.

3. Louis ED, Gerbin M, Galecki M. Essential tremor 10, 20,30, 40: clinical snapshots of the disease by decade of duration. Eur J Neurol 2013;20: 949-54. doi: 10.1111/ene.12123.

4. Prasad S, Bhalsing KS, Hunjhunwala K, Lenka A, Binu VS, Pal PK. Phenotypic variability of essential tremor based on the age at onset. Canadian J Neurol Sci 2019;46:192-8. doi: 10.1017/ cjn.2018.384.

5. Taylor GS. Essential tremor: a review. 2021. Honors Projects. 814

6. Jiménez Jiménez F, Alonso-Navarro H, GarcíaMartín E, Lorenzo-Betancor O, Pastor P, Agúndez JAG. Update on genetics of essential tremor. Acta Neurol Scand 2013;128:359-71. doi: 10.1111/ ane.12148.

7. Louis ED, McCreary M. How common is essential tremor? Update on the worldwide prevalence of essential tremor. Tremor Other Hyperkinetic Movements 2021;11:28. doi: 10.5334/tohm.632.

8. Kuhlenbäumer GF, Hopfner F, Deuschl G. Genetics of essential tremor: meta-analysis and review. Neurology 2014;82:1000-7. doi: 10.1212/WNL. 0000000000000211.

9. Louis ED, Babij R, Ma K, Cortés E, Vonsattel JPG. Essential tremor followed by progressive supranuclear palsy: postmortem reports of 11 patients. J Neuropathol Exp Neurol 2013;72:8-17. doi: 10.1097/NEN.0b013e31827ae56e.

10. Kandemir N, Gultekin M, Kara M, et al. Propranolol decreases DRD3 and SLC1A2 gene expression in patients with essential tremor. Univ Med 2020;39:105-12. doi: 10.18051/UnivMed.2020. v39.10.

11. Tio M, Tan EK. Genetics of essential tremor. Parkinsonism Relat Disord 2016;22 Suppl 1:S176S8. doi: 10.1016/j.parkreldis.2015.09.022.

12. Ondo WG. Current and emerging treatments of essential tremor. Neurol Clin 2020;38:309-23. doi: 10.1016/j.ncl.2020.01.002.

13. Morales J, Li L, Fattah FJ, et al. Review of poly (ADP-ribose) polymerase (PARP) mechanisms of action and rationale for targeting in cancer and other diseases. Crit Rev Eukaryot Gene Expr 2014; 24:15-28. doi: 10.1615/critreveukaryotgeneexpr. 2013006875.
14. Swindall AF, Stanley JA, Yang ES. PARP-1: friend or foe of DNA damage and repair in tumorigenesis? Cancers 2013;5:943-58. doi: 10.3390/cancers503094.3

15. Hoch NC, Polo LM. ADP-ribosylation: from molecular mechanisms to human disease. Genet Mol Biol 2020;43(1 Suppl 1):e20190075. doi: 10.1590/1678-4685-GMB-2019-0075.

16. Zhou T, Pan F, Cao Y, et al. R152C DNA Pol $\beta$ mutation impairs base excision repair and induces cellular transformation. Oncotarget 2016;7:690215. doi: 10.18632/oncotarget.6849.

17. Fahn S, Tolosa E, Marín C. Clinical rating scale for tremor. In: Jankovic J, Tolosa E, editors. Parkinson's disease and movement disorders. $2^{\text {nd }}$. ed. Baltimore: Williams \& Wilkins; 1993.p.225-3.

18. Cersonsky TEK, Kellner S, Morganet S, et al. Demoralization in essential tremor: prevalence, clinical correlates, and dissociation from tremor severity. CNS Spectr 2020;25:16-23. doi: 10.1017/ S1092852918001633.

19. Alonso-Navarro H, García-Martín E, Agúndez JAG, Jiménez-Jiménez FJ. Association between restless legs syndrome and other movement disorders. Neurology 2019;92:948-64. doi: 10.1212/ WNL.0000000000007500.

20. Diez-Fairen M, Bandres-Ciga S, Houle G, et al. Genome-wide estimates of heritability and genetic correlations in essential tremor. Parkinsonism Relat Disord 2019;64:262-7. doi: 10.1016/j. parkreldis.2019.05.002.

21. Hopfner F, Helmich RC. The etiology of essential tremor: genes versus environent. Parkinsonism Relat Disord 2018;46 Suppl 1:S92-S6. doi: 10.1016/ j.parkreldis.2017.07.014.

22. Jiménez-Jiménez FJ, Alonso-Navarro H, GarcíaMartín E. Álvarez I, Pastor P, Agúndez JAG. Genomic markers for essential tremor. Pharmaceuticals 2021;14:516. https://doi.org/ 10.3390/ph14060516J.

23. Liao C, Sarayloo F, Rochefort D, et al. Multiomics analyses identify genes and pathways relevant to essential tremor. Mov Disord 2020;35:1153-62. doi: $10.1002 / \mathrm{mds} .28031$.

24. Shanker V. Essential tremor: diagnosis and management. BMJ 2019;366:14485. doi: 10.1136/ bmj.14485.

25. Hopfner F, Höglinger GU, Kuhlenbäumer G, et al. $\beta$-adrenoreceptors and the risk of Parkinson's disease. Lancet Neurology 2020;19:247-54. doi: 10.1016/S1474-4422(19)30400-4.

26. Fatokun AA, Dawson VL, Dawson TM. Parthanatos: mitochondrial linked mechanisms and therapeutic opportunities. Br J Pharmacol 2014;71:2000-16. doi: 10.1111/bph.12416. 
27. Maynard S, Fang EF, Scheibye-Knudsen M, Croteau DL, Bohr VA. DNA damage, DNA repair, aging, and neurodegeneration. Cold Spring Harb Perspect Med 2015;18:a025130. doi: 10.1101/ cshperspect.a025130.

28. Fang EF, Scheibye-Knudsen M, Brace LE, et al. Defective mitophagy in XPA via PARP-1 hyperactivation and NAD $(+) / S I R T 1$ reduction. Cell 2014;157:882-96. doi: 10.1016/j.cell.2014. 03.026 .

29. Anandhan A, Jacome MS, Lei S, et al. Metabolic dysfunction in Parkinson's disease: bioenergetics, redox homeostasis and central carbon etabolism. Brain Res Bull 2017;133:12-30. doi: 10.1016/j.brainresbull.2017.03.009.

30. Brighina L, Rivab C Bertola F,et al. Association analysis of PARP1 polymorphisms with Parkinson's disease. Parkinsonism Rel Disord 2011;17:701-4. https://doi.org/10.1016/j.parkreldis. 2011.06.022.

31. Alemasova EE, Lavrik OI. Poly(ADP-ribosyl)ation by PARP1: reaction mechanism and regulatory proteins. Nucleic Acids Res 2019;47:3811-27. doi: 10.1093/nar/gkz120.

32. Adamczyk A, Kaźmierczak A, Czapski GA, Strosznajder JB. Alpha-synuclein induced cell death in mouse hippocampal (HT22) cells is mediated by nitric oxide-dependent activation of caspase-3. FEBS Lett 2010;584:3504-8. doi: 10.1016/j.febslet.2010.07.019.

33. Kamal SJ, Khadhim HM. Effects of Irbesartan in induced Parkinson's disease in mice. Int J Pharmaceut Qual Assur 2021;12:31-9. doi: 10.25258/ijpqa.12.1.5.
34. Renani PG, Taheri F, Rostami D, et al. Involvement of aberrant regulation of epigenetic mechanisms in the pathogenesis of Parkinson's disease and epigenetic-based therapies. J Cell Physiol 2019; 234:19307-19. doi: 10.1002/jcp.28622.

35. Broniowska KA, Diers AR, Corbett JA, Hogg N. Effect of nitric oxide on naphthoquinone toxicity in endothelial cells: role of bioenergetic dysfunction and poly (ADP-ribose) polymerase activation. Biochemistry 2013;52:4364-72. doi: 10.1021/bi400342t.

36. Maciag AE, Holland RJ, Kim Y, et al. Nitric oxide (NO) releasing poly ADP-ribose polymerase 1 (PARP-1) inhibitors targeted to glutathione Stransferase P1-overexpressing cancer cells. J Med Chem 2014;57:2292-302. doi: 10.1021/ jm401550d.

37. Krokan HE, Bjøras M. Base excision repair. Cold Spring Harb Perspect Biol 2013;5:a012583. doi: 10.1101/cshperspect.a012583.

38. Beard WA, Wilson SH. DNA polymerase beta and other gap-filling enzymes in mammalian base excision repair. Enzymes 2019;45:1-26. doi: 10.1016/bs.enz.2019.08.002.

39. Wang H, Chen Y, Chen J, Cell cycle regulation of DNA polymerase beta in rotenone-based Parkinson's disease models. PLoS One 2014;9: e109697. doi: 10.1371/journal.pone.0109697. 